\title{
Postoperative kidney oxygen saturation as a novel marker for acute kidney injury after adult cardiac surgery
}

Christian Ortega-Loubon, MD, MEd, ${ }^{\text {a }}$ Manuel Fernández-Molina, MD, ${ }^{a}$ Inmaculada Fierro, $\mathrm{PhD},{ }^{\mathrm{b}}$ Pablo Jorge-Monjas, MD, $\mathrm{PhD},{ }^{c}$ Yolanda Carrascal, $\mathrm{MD}, \mathrm{PhD},{ }^{\mathrm{a}}$ José I. Gómez-Herreras, $\mathrm{MD}, \mathrm{PhD},{ }^{\mathrm{c}}$ and Eduardo Tamayo, $\mathrm{MD}, \mathrm{PhD}^{\mathrm{c}}$

\begin{abstract}
Objective: Acute kidney injury is a severe complication and one of the stronger risk factors for death in patients undergoing cardiac surgery. The relationship between postoperative brain oxygen saturation and kidney oxygen saturation with acute kidney injury in adults undergoing cardiac surgery has not been determined. We designed a single-center prospective study to determine if the continuous monitoring of postoperative brain oxygen saturation and kidney oxygen saturation could predict postoperative acute kidney injury.
\end{abstract}

Methods: We conducted a prospective open cohort study from January to September 2017. The primary outcome was postoperative acute kidney injury using the Kidney Disease: Improving Global Outcomes criteria. Brain oxygen saturation and kidney oxygen saturation, the metrics of which were area measurements (\%-min), were recorded during the surgery and the first 48 hours after the cardiac procedure. Receiver operating characteristic curve analysis was used to evaluate the predictive power of kidney oxygen saturation for acute kidney injury.

Results: A total of 121 consecutive patients were enrolled. Thirty-five patients $(28.9 \%)$ developed acute kidney injury. Brain oxygen saturation showed no statistical difference in both groups; however, kidney oxygen saturation was related to acute kidney injury $(P=.001)$. Receiver operating characteristic curve analysis showed that kidney oxygen saturation could predict the risk of acute kidney injury. Kidney oxygen saturation less than $65 \%$ (area under the curve-receiver operating characteristic, $0.679 \pm 0.054,95 \%$ confidence interval, $0.573-0.785, P=.002$ ) and $20 \%$ decrease from baseline (area under the curve-receiver operating characteristic, $0.639 \pm 0.059,95 \%$ confidence interval, $0.523-0.755, P=.019)$ showed the better performance, respectively.

Conclusions: Postoperative kidney oxygen saturation is related to the development of cardiac surgery-associated acute kidney injury. Continuous kidney saturation monitoring might be a promising, noninvasive tool for predicting acute kidney injury during the postoperative period for adult patients after cardiac surgery. (J Thorac Cardiovasc Surg 2019;157:2340-51)

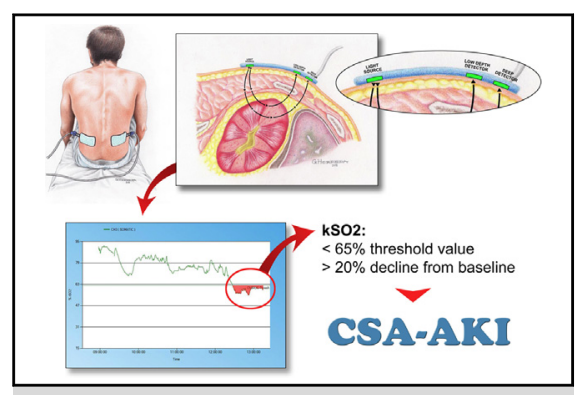

$\mathrm{KSO}_{2}$ desaturation significantly increases the risk of CSA-AKI.

\section{Central Message}

Postoperative kidney oxygen desaturation is related to CSA-AKI. Its continuous monitoring could be a real-time tool for predicting AKI.

\section{Perspective}

Absolute kidney oxygen desaturations less than $65 \%$ or decreases greater than $20 \%$ from baseline during the postoperative period are novel markers for developing postoperative CSAAKI. Continuous kidney oxygen saturation monitoring could be a real-time, noninvasive strategy to improve AKI detection, allowing prompt appropriate responses.

See Commentaries on pages 2352 and 2354.

\footnotetext{
From the ${ }^{\mathrm{a}}$ Department of Cardiac Surgery and ${ }^{\mathrm{c}}$ Anesthesiology and Critical Care, Clinic University Hospital of Valladolid, Valladolid, Spain; and ${ }^{\mathrm{b}}$ Department of Health Science, Universidad Europea Miguel de Cervantes, Valladolid, Spain. Support was provided solely from institutional and/or departmental sources.

Received for publication Feb 22, 2018; revisions received Sept 6, 2018; accepted for publication Sept 28, 2018; available ahead of print Nov 17, 2018.

Address for reprints: Christian Ortega-Loubon, MD, MEd, Cardiac Surgery Department, Clinic University Hospital of Valladolid, Ramón y Cajal Ave, Valladolid 47003, Spain (E-mail: christlord26@gmail.com).

0022-5223/\$36.00

Copyright (c) 2018 by The American Association for Thoracic Surgery

https://doi.org/10.1016/j.jtcvs.2018.09.115
}

Acute kidney injury (AKI) is a severe complication and one of the stronger risk factors for death in patients undergoing cardiac surgery, with an incidence ranging from $8.9 \%$ to $42.5 \%$, depending on the definition. ${ }^{1}$ It is a frequent and

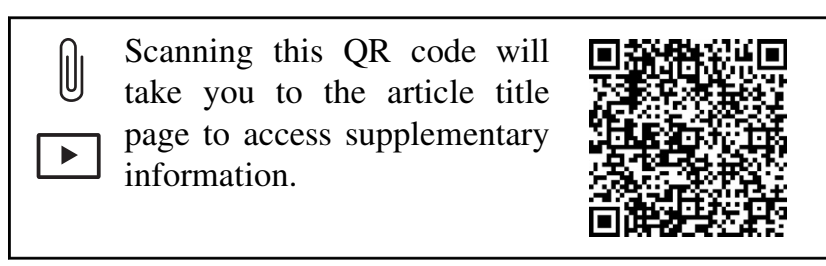




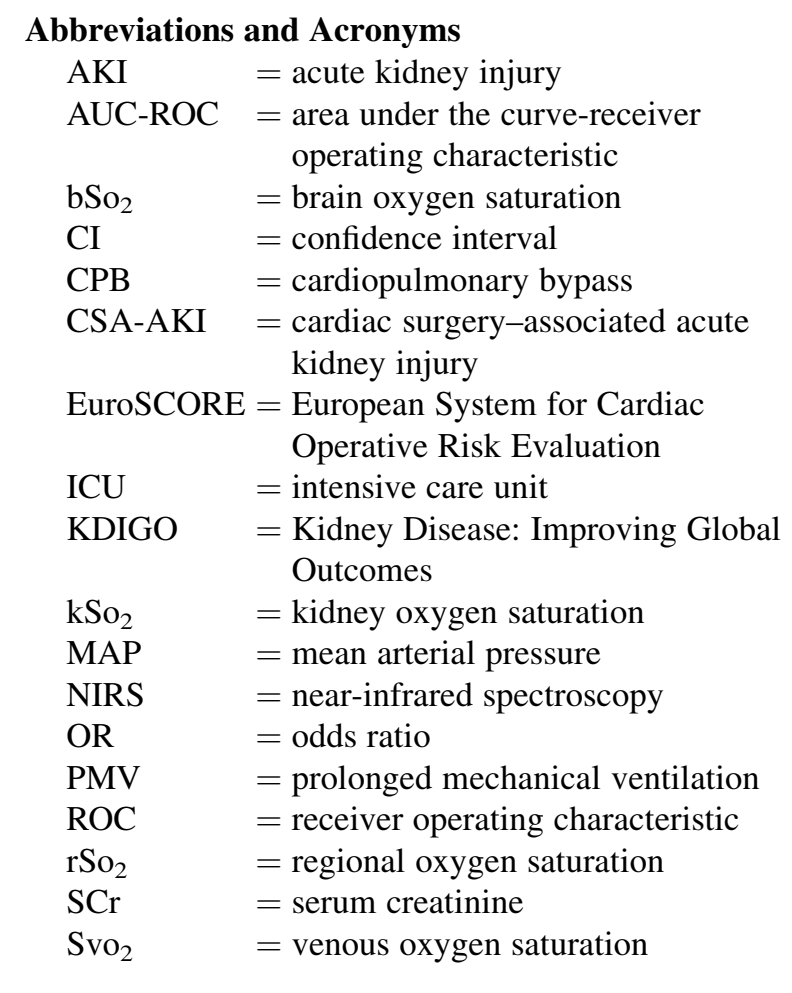

severe complication of cardiac surgery, and cardiac surgery itself is the second most common cause of AKI in the intensive care unit (ICU). This association is what led to the term "cardiac surgery-associated acute kidney injury" (CSAAKI). ${ }^{2}$

Many efforts have been directed to the development and validation of novel predictor scales. However, all of them have limitations. Current predictor scales are based on serum creatinine ( $\mathrm{SCr}$ ), which is affected by factors such as age, sex, ethnicity, muscle mass, and intravascular volume, regardless of renal function. Furthermore, SCr does not increase until $50 \%$ of the renal function is lost, which can limit and even delay its detection. ${ }^{3}$ Several biomarkers, such as neutrophil gelatinase-associated lipocalin, cystatin C, kidney injury molecule 1 , and interleukin 18 , have been shown to facilitate early diagnosis. ${ }^{4}$ Nevertheless, their levels increase 2 to 6 hours after surgery. ${ }^{5}$ Although these biomarkers may be the most sensitive means of CSA-AKI early detection, they cannot be detected faster or measured continuously to monitor renal function in real time, which is needed to optimize renal function. It is essential to identify these high-risk patients early to allow prompt and appropriate responses. ${ }^{6}$ The ideal measure would be a continuous, noninvasive monitor to observe any immediate alterations related to the higher incidence of CSA-AKI, thus allowing clinicians a chance to avoid it.

Near-infrared spectroscopy (NIRS) is a noninvasive technique that continuously measures the difference between oxygenated and deoxygenated hemoglobin within a regional tissue area, obtaining the regional oxygen saturation $\left(\mathrm{rSO}_{2}\right){ }^{7}$ Different from $\mathrm{SCr}$, NIRS measurement is limited by its depth of penetration. Other factors potentially impairing NIRS measurement are high concentration of conjugated bilirubin, amount of skin pigment, and any external source of light that may influence or disturb the signal detection. ${ }^{8}$ In cardiac surgery, NIRS is usually used to monitor brain oxygen saturation $\left(\mathrm{bSo}_{2}\right)$. Intraoperative brain desaturation is related to an increased risk of cognitive decline $^{9}$ and intraoperative kidney oxygen desaturation to postoperative AKI in adult patients. ${ }^{10}$ However, there may be no significant changes in $\mathrm{bSo}_{2}$ with significant decreases observed in kidney oxygen saturation $\left(\mathrm{kSo}_{2}\right)$ or other somatic $\mathrm{rSo}_{2}$ during crossclamp. ${ }^{11}$ The relationship between postoperative $\mathrm{kSO}_{2}$ and $\mathrm{AKI}$ in adults undergoing cardiac surgery has not been determined, because most studies have only focused on intraoperative period monitoring, leaving the direct influence in the postoperative time span unknown.

We hypothesize that postoperative $\mathrm{kSo}_{2}$ may be most closely related to postoperative CSA-AKI than $\mathrm{bSo}_{2}$. This study aims to determine if the continuous monitoring of postoperative $\mathrm{bSo}_{2}$ and $\mathrm{kSO}_{2}$ can predict and serve as a parameter for the prevention of postoperative CSA-AKI.

\section{MATERIAL AND METHODS \\ Study Design}

This study (identification number PI 17584, CINV 17-03) received full approval by both the local Institutional Research Review Committee and the Clinical Research Ethic Committee; the latter consists of 15 members. They waived the patient informed consent requirement; however, all patients gave their written consent for storing their data in an anymous form in the hospital database for scientific purposes at the time of hospital admission, in accordance with the Spanish law regulating personal privacy matters.

We designed a prospective open cohort study to assess if continuous brain and kidney NIRS records during the surgery and the first 48 hours of the postoperative period correlate with CSA-AKI using the Kidney Disease: Improving Global Outcomes (KDIGO) criteria (ie, an increase in $\mathrm{SCr}$ by $\geq 0.3 \mathrm{mg} / \mathrm{dL}$ or greater within 48 hours; or an increase in $\mathrm{SCr}$ to $\geq 1.5$ times baseline, within 7 days after surgery). ${ }^{12}$ We used the $\mathrm{SCr}$ levels obtained at the hospital admission evaluation, and the peak SCr levels reached up to 7 days of the postoperative course.

This study was carried out from January to September 2017 in the Clinic University Hospital of Valladolid, Spain.

\section{Near-Infrared Spectroscopy Measurements}

$\mathrm{bSo}_{2}$ and $\mathrm{kSO}_{2}$ were monitored with NIRS (INVOS 5100C; Medtronic, Inc, Minneapolis, Minn) during the surgery and the first 48 hours after cardiac procedure. Thus, the NIRS monitoring phase for CSA-AKI lasts 48 hours after cardiac surgery, but the observation phase spanned 7 days after the cardiac intervention. Before the induction of anesthesia, NIRS sensors were placed on both sides of the forehead and on both sides of the flank area that overlies the kidney previously marked under ultrasound guidance to monitor the $\mathrm{kSO}_{2} .{ }^{10}$

A baseline $\mathrm{rSO}_{2}$ value was determined in each patient before the surgery without the administration of any anesthetic drugs or supplemental oxygen 
that could modify its real value. Both $\mathrm{bSo}_{2}$ and $\mathrm{kSO}_{2}$ were recorded every 30 seconds during the intraoperative and postoperative period, and up to 48 hours after cardiac surgery. These values represent the intraoperative and postoperative NIRS measurements.

Recorded $\mathrm{rSO}_{2}$ data were analyzed using INVOS Analytics Tools software (Medtronic, Inc). The operative variables were any $\mathrm{bSO}_{2}$ and $\mathrm{kSO}_{2}$ desaturations below $70 \%, 65 \%, 60 \%$, and $55 \%$ threshold values, as well as declines of $\mathrm{bSO}_{2}$ and $\mathrm{kSO}_{2}$ greater than $5 \%, 10 \%, 15 \%, 20 \%, 25 \%$, and $30 \%$ from the patient-specific baseline value. We analyzed the area (\%-min), which accounts for both the intensity of the desaturation from a defined value (from a fixed threshold value or the patient-specific baseline value) and the duration of that desaturation.

No intervention was attempted when desaturations were recorded. However, in cases in which there were intense $\mathrm{bSo}_{2}$ and $\mathrm{kSO}_{2}$ desaturations, appropriate measures were taken to optimize the hemodynamic data of the patients.

\section{Study Patients}

A total of 121 consecutive patients scheduled for elective cardiac surgery under cardiopulmonary bypass (CPB) were enrolled. Exclusion criteria were prior renal insufficiency, off-pump surgery, urgent/emergency surgery, kidney transplant, and kidney depth $4 \mathrm{~cm}$ or greater from the skin. ${ }^{10}$

\section{Study Variables}

Demographic data included age, sex, body mass index, diabetes mellitus, hypertension, peripheral vascular disease, left ventricle ejection fraction, logistic European System for Cardiac Operative Risk Evaluation (EuroSCORE), kidney depth, baseline $\mathrm{bSo}_{2}, \mathrm{kSO}_{2}$, preoperative hemoglobin, preoperative $\mathrm{SCr}$, and estimated glomerular filtration rate. The estimated glomerular filtration rate was calculated using the Chronic Kidney Disease Epidemiology Collaboration formula. Laboratory postoperative parameters were collected during the following stages of the study: ICU admission, 6 hours, 12 hours, 24 hours, and 48 hours postoperatively.

The primary outcome was any degree of postoperative CSA-AKI defined by the KDIGO criteria. Secondary outcomes included major adverse cardiac and noncardiac events, such as reoperation, sepsis, low cardiac output, vasoplegia, pneumonia, prolonged mechanical ventilation (PMV), excessive bleeding, massive transfusion, mortality, ICU stay, and length of hospital stay.

\section{Definitions}

Patients with a cardiac index less than $2.2 \mu \mathrm{L} / \mathrm{min} / \mathrm{m}^{2}$ were deemed to have low cardiac output. Vasoplegia was defined as a normal cardiac index with a requirement of 1 or 2 vasopressors to maintain a mean arterial pressure (MAP) of approximately $70 \mathrm{~mm} \mathrm{Hg}{ }^{13}$ PMV was considered greater than 48 hours. ${ }^{14}$ Excessive bleeding was considered if more than $1000 \mathrm{~mL}$ in the first 12 to 24 postoperative hours was lost, and massive transfusion meant more than 10 units of allogeneic blood products were required. ${ }^{15}$

\section{Statistical Analysis}

Categoric variables are reported as percentages, and continuous variables are reported as the mean \pm standard deviation or median (interquartile range) as appropriate, attending normal distribution. The assumption of normality was evaluated using the Shapiro-Wilk or Kolmogorov-Smirnov test.

The associations between CSA-AKI and other variables were identified using the chi-square or Fisher exact test (when the expected frequencies were $<5$ ) for categoric variables, and the Student $t$ test or the MannWhitney $U$ test was used for continuous variables according to normality criteria.

Variables were included in univariate logistic regression analysis, and bootstrapping was performed to derive $95 \%$ confidence intervals (CIs) for estimates. A multivariable logistic regression model was developed using a stepwise selection of predictors for CSA-AKI. The logistic regression was repeated with the variables that had been significant in the previous analysis. Variables selection and weighting were further checked by bootstrapping sampling. Internal validation processes were performed using bootstrapping, random sampling with 100 -fold cross-validation (Tables E1-E3).

Accuracy was measured by the area under the curve-receiver operating characteristic (AUC-ROC) curve analysis. To determine the best predictive threshold value of the AUC-ROC curve, the analysis was performed at every threshold value and every decline from the baseline value.

Correlations were used to determine the relationship between $\mathrm{bSo}_{2}$ and $\mathrm{kSO}_{2}$. The odds ratio (OR) with $95 \% \mathrm{CI}$ and $P$ values were reported. Data were analyzed using IBM SPSS Statistics for Windows version 24.0 software (IBM Corp, Armonk, NY).

\section{RESULTS}

A total of 35 patients $(28.9 \%)$ developed CSA-AKI. The median age was 70 years (interquartile range, 64-75), and $47.1 \%$ of the patients $(n=57)$ were female. Preoperative, intraoperative, and postoperative data are listed in Table 1 , and postoperative outcomes are shown in Table 2.

Spearman's rho correlations were used to determine the relationship between $\mathrm{bSo}_{2}$ and $\mathrm{kSo}_{2}$ (not normally distributed variables). $\mathrm{bSo}_{2}$ from both left and right forehead sites were highly correlated $(P=.827)$, similarly to $\mathrm{kSo}_{2}$ from both left and right flank areas $(P=.814)$. However, $\mathrm{bSo}_{2}$ and $\mathrm{kSo}_{2}$ were weakly correlated from each other at any side $\left(\mathrm{bSo}_{2} \mathrm{~L} / \mathrm{kSo}_{2} \mathrm{~L}, P=.375 ; \mathrm{bSo}_{2} \mathrm{~L} / \mathrm{kSo}_{2} \mathrm{R}, P=.401\right.$; $\mathrm{bSo}_{2} \mathrm{R} / \mathrm{kSo}_{2} \mathrm{~L}, P=.321 ; \mathrm{bSo}_{2} \mathrm{R} / \mathrm{kSo}_{2} \mathrm{R}, P=.403$; Table 3$)$.

\section{Kidney Oxygen Saturation Analysis}

Table 4 reports the area analysis for $\mathrm{bSo}_{2}$ and $\mathrm{kSO}_{2}$ during the intraoperative and postoperative periods.

Concerning $\mathrm{kSo}_{2}$ during the postoperative extent, patients with CSA-AKI had higher median desaturations at each threshold value (ie, $<70 \%,<65 \%,<60 \%$, and $<55 \%$ ) and higher decreases from baseline value compared with patients without CSA-AKI (Table 3). $\mathrm{kSo}_{2}$ less than $70 \%, 65 \%, 60 \%$, and $55 \%$ was related to CSA-AKI $(P=.005, P=.001, P=.001$, and $P=.013$, respectively). The declines of $\mathrm{kSO}_{2}$ greater than $15 \%, 20 \%, 25 \%$, and $30 \%$ from baseline value were significantly related to CSA-AKI during the postoperative course $(P=.050$, $P=.013, \mathrm{P}<.001$, and $P=.009$, respectively; Table 4).

The univariate analysis showed that a postoperative decrease in $\mathrm{kSO}_{2}$ greater than $25 \%$ from the baseline value was related to CSA-AKI (OR, 3.90; 95\% CI, 1.68-9.02). Although multivariable analysis showed a decrease in $\mathrm{kSO}_{2}$ greater than $20 \%$ from baseline as a risk of CSAAKI (2.707; 95\% CI, 1.07-6.81), the postoperative decline in $\mathrm{kSo}_{2}$ greater than $25 \%$ from baseline remained the strongest risk factor with the highest OR of any value (3.60; CI, 1.46-8.85; Table 5).

The probability of postoperative CSA-AKI was significantly higher in patients with absolute $\mathrm{kSo}_{2}$ desaturations 
TABLE 1. Preoperative, intraoperative, and postoperative characteristics according to the presence of cardiac surgery-associated acute kidney injury

\begin{tabular}{|c|c|c|c|c|}
\hline Variables & Overall (n 121) & Non-CSA-AKI (86) & CSA-AKI (35) & $P$ value \\
\hline \multicolumn{5}{|l|}{ Preoperative data } \\
\hline \multicolumn{5}{|l|}{ Demographic data } \\
\hline Age, y, median (IQR) & $70(64-75)$ & $68(61-74)$ & $72(67-76)$ & .047 \\
\hline Female & $57(47.1)$ & $41(47.7)$ & $16(45.7)$ & .845 \\
\hline $\mathrm{BMI}, \mathrm{kg} / \mathrm{m}^{2}$, mean $\pm \mathrm{SD}$ & $27.0 \pm 3.7$ & $26.9 \pm 3.8$ & $27.2 \pm 3.5$ & .624 \\
\hline EuroSCORE II, median (IQR) & $2.9(1.5-4.7)$ & $2.4(1.3-4.1)$ & $3.7(2.4-5.9)$ & .004 \\
\hline \multicolumn{5}{|l|}{ Comorbidities } \\
\hline Smoker & $49(40.5)$ & $32(37.2)$ & $17(48.6)$ & .248 \\
\hline Current smoker & $11(9.1)$ & $9(10.5)$ & $2(5.7)$ & $.508^{*}$ \\
\hline Hypertension & $89(73.6)$ & $60(69.8)$ & $29(82.9)$ & .139 \\
\hline Diabetes mellitus & $24(19.8)$ & $15(17.4)$ & $9(25.7)$ & .301 \\
\hline Dyslipidemia & $72(59.5)$ & $49(57.0)$ & $23(65.7)$ & .375 \\
\hline COPD & $12(9.9)$ & $4(4.7)$ & $8(22.9)$ & $.005^{*}$ \\
\hline Coronary artery disease & $47(38.8)$ & $29(33.7)$ & $18(51.4)$ & .070 \\
\hline Valve disease & $102(84.3)$ & $71(82.6)$ & $31(88.6)$ & .410 \\
\hline Peripheral vascular disease & $5(4.1)$ & $3(3.5)$ & $2(5.7)$ & $.626^{*}$ \\
\hline Prior cardiac surgery & $5(4.1)$ & $4(4.7)$ & $1(2.9)$ & $1.000^{*}$ \\
\hline Prior stroke & $7(5.8)$ & $3(3.5)$ & $4(11.4)$ & $.106^{*}$ \\
\hline $\mathrm{AF}$ & $50(41.3)$ & $32(37.2)$ & $18(51.4)$ & .150 \\
\hline NYHA 3 & $52(43.0)$ & $40(46.5)$ & $12(34.3)$ & $.099 *$ \\
\hline NYHA 4 & $5(4.1)$ & $2(2.3)$ & $3(8.6)$ & \\
\hline Left ventricular dysfunction (LVEF $<50 \%$ ) & $22(18.2)$ & $15(17.4)$ & $7(20.0)$ & .741 \\
\hline \multicolumn{5}{|l|}{ Intraoperative data } \\
\hline CPB time, min, median (IQR) & $109.0(90.5-139.0)$ & $96.0(81.8-130.5)$ & $134.0(120.0-170.0)$ & $<.001$ \\
\hline Aortic crossclamp time, min, median (IQR) & $80.0(63.0-110.5)$ & $74.5(60.0-103)$ & $105.0(85.0-119.0)$ & .001 \\
\hline CABG & $17(14.0)$ & $13(15.1)$ & $4(11.4)$ & $.775^{*}$ \\
\hline Valve surgery & $97(80.2)$ & $68(79.1)$ & $29(82.9)$ & .212 \\
\hline $\mathrm{CABG}+$ valve & $19(15.7)$ & $10(11.6)$ & $9(25.7)$ & .050 \\
\hline Other surgery & $11(9.1)$ & $7(8.1)$ & $4(11.4)$ & $1.000^{*}$ \\
\hline MAP, mm Hg, median (IQR) & $67.4(61.5-71.6)$ & $67.4(61.5-71.2)$ & $67.4(61.5-72.3)$ & .819 \\
\hline Peak lactate, mmol/L, median (IQR) & $4.4(2.8-26.0)$ & $4.3(2.7-25.0)$ & $14.0(2.8-30.0)$ & .256 \\
\hline \multicolumn{5}{|l|}{ Postoperative data } \\
\hline Minimum cardiac output, $\mathrm{L} / \mathrm{min} / \mathrm{m}^{2}$, median (IQR) & $2.0(1.8-2.3)$ & $2.1(1.9-2.3)$ & $1.9(1.7-2.2)$ & .187 \\
\hline $\mathrm{SvO}_{2}$ (admission), $\%$, mean $\pm \mathrm{SD}$ & $75.2 \pm 10.3$ & $75.4 \pm 10.3$ & $74.5 \pm 10.4$ & .668 \\
\hline $\mathrm{SvO}_{2} 6 \mathrm{~h}$, mean $\pm \mathrm{SD}$ & $72.2 \pm 8.9$ & $72.3 \pm 8.7$ & $72.0 \pm 9.6$ & .838 \\
\hline $\mathrm{Svo}_{2} 12 \mathrm{~h}$, mean $\pm \mathrm{SD}$ & $67.2 \pm 8.9$ & $68.8 \pm 8.6$ & $64.3 \pm 12.8$ & .023 \\
\hline $\mathrm{Svo}_{2} 24 \mathrm{~h}$, mean $\pm \mathrm{SD}$ & $65.7 \pm 9.5$ & $67.1 \pm 9.2$ & $62.5 \pm 9.7$ & .017 \\
\hline VIS, median (IQR) & $3.0(0.0-10.5)$ & $0.0(0.0-6.3)$ & $14.0(0.0-45.0)$ & $<.001$ \\
\hline Oliguria, $<30 \mathrm{~mL} / \mathrm{h}^{-1}$ & $18(14.9)$ & $1(1.2)$ & $17(48.6)$ & $<.001$ \\
\hline
\end{tabular}

CSA-AKI, Cardiac surgery-associated acute kidney injury; IQR, interquartile range; BMI, body mass index; SD, standard deviation; EuroSCORE, European System for Cardiac Operative Risk Evaluation; $C O P D$, chronic obstructive pulmonary disease; $A F$, atrial fibrillation; NYHA, New York Heart Association; $L V E F$, left ventricular ejection fraction; $C P B$, cardiopulmonary bypass; $C A B G$, coronary artery bypass graft; $M A P$, mean arterial pressure; VIS, vasoactive inotrope score. *Fisher exact test.

less than $65 \%, 60 \%$, and $55 \%(P=.004, P=.020$, $P=.039$, respectively), and declines of $\mathrm{kSo}_{2}$ greater than $20 \%, 25 \%$, and $30 \%$ from baseline $(P=.036, P=.005$, $P=.036$, respectively; Figure 1). Fifty percent of the patients who developed postoperative CSA-AKI underwent a $\mathrm{kSO}_{2}$ decline greater than $20 \%$ from the baseline value for more than 2.2 minutes.

ROC curve analysis showed that $\mathrm{kSO}_{2}$ could predict the risk of AKI. $\mathrm{kSO}_{2}$ less than $65 \%$ (AUC-ROC, $0.679 \pm 0.054 ; 95 \% \mathrm{CI}, 0.573-0.785 ; P=.002)$ and $\mathrm{a}$
$20 \%$ decline from baseline in $\mathrm{kSo}_{2}$ (AUC-ROC, $0.639 \pm 0.059 ; 95 \%$ CI, $0.523-0.755 ; P=.019)$ had the better performance, respectively (Table 6). Receiver operating characteristic curve figures are available in Figures E1 and E2.

\section{Brain Oxygen Saturation Analysis}

There were no statistically significant differences between any $\mathrm{bSo}_{2}$ or $\mathrm{kSO}_{2}$ threshold values, or any decline of $\mathrm{bSo}_{2}$ or $\mathrm{kSo}_{2}$ from baseline and postoperative CSA-AKI 
TABLE 2. Patient postoperative outcomes according to the presence of cardiac surgery-associated acute kidney injury

\begin{tabular}{|c|c|c|c|c|}
\hline Variables & n $121(\%)$ & Non-AKI (86) & AKI (35) & $P$ value \\
\hline Reoperation & $11(9.1)$ & $3(3.5)$ & $8(22.9)$ & $.002 *$ \\
\hline Delirium & 14 (11.6) & $6(7.0)$ & $8(22.9)$ & $.024 *$ \\
\hline Convulsion & $4(3.3)$ & $2(2.3)$ & $2(5.7)$ & $.578^{*}$ \\
\hline Sepsis & $3(2.5)$ & $0(0.0)$ & $3(8.6)$ & $.023^{*}$ \\
\hline Infection & $18(14.9)$ & $9(10.5)$ & $9(25.7)$ & .033 \\
\hline Arrhythmia & $51(42.1)$ & $32(37.2)$ & $19(54.3)$ & .085 \\
\hline Circulatory arrest & $3(2.5)$ & $0(0.0)$ & $3(8.6)$ & $.023^{*}$ \\
\hline Low cardiac output & $34(28.1)$ & $17(19.8)$ & $17(48.6)$ & .001 \\
\hline Vasoplegia & $13(10.7)$ & $4(4.7)$ & $9(25.7)$ & $.002 *$ \\
\hline Pneumonia & $6(5.0)$ & $1(1.2)$ & $5(14.3)$ & $.008 *$ \\
\hline PMV & $9(7.5)$ & $1(1.2)$ & $8(23.5)$ & $.000^{*}$ \\
\hline Hepatic dysfunction & $19(15.7)$ & $9(10.5)$ & $10(28.6)$ & .013 \\
\hline Massive transfusion & $16(13.2)$ & $5(5.8)$ & $11(31.4)$ & $<.001^{*}$ \\
\hline 30-d mortality & $1(50.0)$ & $0(0.0)$ & $1(100.0)$ & $1.000^{*}$ \\
\hline ICU stay, median (IQR) & $3.0(2.0-4.0)$ & $3.0(2.0-4.0)$ & $4.0(3.0-6.0)$ & $<.001$ \\
\hline Length of hospital stay, median (IQR) & $12.0(9.0-15.0)$ & $11.0(9.0-14.0)$ & $15.0(10.0-24.0)$ & $<.001$ \\
\hline
\end{tabular}

$A K I$, Acute kidney injury; $P M V$, prolonged mechanical ventilation; $I C U$, intensive care unit; $I Q R$, interquartile range. *Fisher exact test.

during the intraoperative term. Severe absolute $\mathrm{KSo}_{2}$ desaturations less than $55 \%$ or declines of $\mathrm{kSo}_{2}$ greater than $30 \%$ from baseline were far less common than absolute $\mathrm{bSo}_{2} \mathrm{de}-$ saturations and declines of $\mathrm{bSo}_{2}$ from baselines during both the surgery and the postoperative phases. Regarding the postoperative stage, patients with CSA-AKI had higher median absolute $\mathrm{bSo}_{2}$ desaturations at every threshold value and higher declines of $\mathrm{bSo}_{2}$ from baseline value compared with patients without CSA-AKI. However, there were no statistically significant differences between any $\mathrm{bSo}_{2}$ threshold value or any decline of $\mathrm{bSo}_{2}$ from baseline and postoperative CSA-AKI (Table 4).

Patients with CSA-AKI were older $(P=.047)$, had higher EuroSCORE $(P=.004)$, had longer CPB time $(P<.001)$, had longer aortic crossclamp time $(P=.001)$, tended to require more vasoactive inotrope support $(P<.001)$, and required more blood transfusions $(P<.001)$. The majority of procedures performed were

TABLE 3. Spearman rho correlations between brain oxygen saturation and kidney oxygen saturation

\begin{tabular}{lcccc}
\hline & $\mathbf{b S o}_{2}$ left & $\mathbf{b S o}_{2}$ right & $\mathbf{k S o}_{2}$ left & $\mathbf{k S o}_{2}$ right \\
\hline $\mathrm{bSo}_{2}$ left & 1.000 & $0.827^{*}$ & $0.375^{*}$ & $0.401^{*}$ \\
$\mathrm{bSo}_{2}$ right & $0.827^{*}$ & 1.000 & $0.321^{*}$ & $0.403^{*}$ \\
$\mathrm{kSo}_{2}$ left & $0.375^{*}$ & $0.321^{*}$ & 1.000 & $0.814^{*}$ \\
$\mathrm{kSo}_{2}$ right & $0.401^{*}$ & $0.403^{*}$ & $0.814^{*}$ & 1.000 \\
\hline
\end{tabular}

$\mathrm{bSo}_{2}$, Brain oxygen saturation; $\mathrm{kSo}_{2}$, kidney oxygen saturation. *Data show statistical significance $P<.001$. replacement valve surgeries $(80.2 \%)$. Nevertheless, there was no statistically significant difference between the procedures performed and CSA-AKI, except for the combination of coronary artery bypass graft and valve replacement surgery $(P=.050)$.

There were significant differences in laboratory findings during different moments of the study (Figure 2). Postoperative hemoglobin values were lower in patients with CSAAKI during the first 6,12 , and 24 hours after surgery $(P=.002, P=.003$, and $P=.018$, respectively). Lactate levels, procalcitonin, and high-sensitivity troponin T levels persisted significantly higher in patients with CSA-AKI during the entire postoperative term $(P<.001)$.

Patients with CSA-AKI had worse secondary outcomes, consisting of oliguria $(P<.001)$, reoperation $(P=.002)$, sepsis $(P=.023)$, low cardiac output $(P=.001)$, vasoplegia $(P=.002)$, PMV $(P=.001)$, excessive bleeding $(P=.002)$, and longer ICU $(P<.001)$ and hospital stays $(P<.001$; Table 2).

\section{DISCUSSION}

There were 4 major findings in this study. First, a decrease in the $\mathrm{kSo}_{2}$ to a value less than $65 \%$ or a decline greater than $20 \%$ from baseline value during the postoperative period is significantly associated with CSA-AKI, as depicted in Figure 3. Second, declines in $\mathrm{bSo}_{2}$ and $\mathrm{kSO}_{2}$ during the intraoperative interval did not correlate with postoperative CSA-AKI. Third, declines in $\mathrm{bSo}_{2}$ during the postoperative term did not correlate with postoperative 
TABLE 4. Comparison of intraoperative and postoperative brain oxygen saturation and kidney oxygen saturation areas (\%-min) below different absolute threshold values and at multiple declines from baseline

\begin{tabular}{|c|c|c|c|c|}
\hline & Overall $(n=121)$ & Non-CSA-AKI (n 86) & CSA-AKI (n 35) & $P$ value \\
\hline \multicolumn{5}{|l|}{ Surgery } \\
\hline \multicolumn{5}{|l|}{$\mathrm{bSo}_{2}$} \\
\hline$<70 \%$ & $2505(1028-4172)$ & $2382(1004-4138)$ & $2650(1317-4172)$ & .653 \\
\hline$<65 \%$ & $1394(434-2744)$ & $1324(329-2895)$ & $1479(622-2743)$ & .910 \\
\hline$<60 \%$ & $537(103-1533)$ & $540(103-1725)$ & $515(203-1502)$ & .995 \\
\hline$<55 \%$ & $125(4-719)$ & $128(1-733)$ & $125(7-719)$ & .805 \\
\hline \multicolumn{5}{|c|}{ Decline from baseline } \\
\hline$>5 \%$ & $1236(400-2624)$ & $1338(432-2634)$ & $995(238-2624)$ & .433 \\
\hline$>10 \%$ & $531(122-1437)$ & $530(142-1487)$ & $551(101-1204)$ & .725 \\
\hline$>15 \%$ & $277(35-1023)$ & $324(38-1156)$ & $199(24-1001)$ & .433 \\
\hline$>20 \%$ & $88(1-555)$ & $140(1-574)$ & $47(1-555)$ & .758 \\
\hline$>25 \%$ & $19(0-248)$ & $22(0-339)$ & $13(0-192)$ & .685 \\
\hline$>30 \%$ & $1(0-90)$ & $1(0-111)$ & $1(0-62)$ & .977 \\
\hline \multicolumn{5}{|l|}{$\mathrm{kSO}_{2}$} \\
\hline$<70 \%$ & $141(1-561)$ & $143(2-528)$ & $139(0-761)$ & .988 \\
\hline$<65 \%$ & $3(0-172)$ & $4(0-184)$ & $0(0-171)$ & .695 \\
\hline$<60 \%$ & $0(0-18)$ & $0(0-24)$ & $0(0-11)$ & 688 \\
\hline$<55 \%$ & $0(0-0)$ & $0(0-0)$ & $0(0-0)$ & .527 \\
\hline \multicolumn{5}{|c|}{ Decline from baseline } \\
\hline$>5 \%$ & $1012(406-1878)$ & $1046(487-1962)$ & 767 (151-1864) & .203 \\
\hline$>10 \%$ & $419(98-1131)$ & $479(115-1151)$ & $329(43-887)$ & .259 \\
\hline$>15 \%$ & $119(4-543)$ & $148(7-630)$ & $67(0-454)$ & .219 \\
\hline$>20 \%$ & $8(0-208)$ & $12(0-223)$ & $2(0-89)$ & .271 \\
\hline$>25 \%$ & $0(0-45)$ & $0(0-81)$ & $0(0-12)$ & .533 \\
\hline$>30 \%$ & $0(0-0)$ & $0(0-1)$ & $0(0-0)$ & .567 \\
\hline \multicolumn{5}{|c|}{ Postoperative } \\
\hline \multicolumn{5}{|l|}{$\mathrm{bSo}_{2}$} \\
\hline$<70 \%$ & $7586(3249-14,736)$ & $7502(277-14033)$ & $7586(3362-15,129)$ & .837 \\
\hline$<65 \%$ & 3355 (735-7034) & $3200(660-6866)$ & $3525(840-8245)$ & .662 \\
\hline$<60 \%$ & $832(71-2748)$ & $722(57-2740)$ & $1384(99-2955)$ & .454 \\
\hline$<55 \%$ & $135(1-968)$ & $107(0-950)$ & $159(11-977)$ & .377 \\
\hline \multicolumn{5}{|c|}{ Decline from baseline } \\
\hline$>5 \%$ & $2380(559-8835)$ & $2436(618-8535)$ & $2138(236-11711)$ & .834 \\
\hline$>10 \%$ & 874 (193-4995) & $870(218-4836)$ & $875(47-6344)$ & .882 \\
\hline$>15 \%$ & $253(16-1945)$ & $231(16-1880)$ & $294(17-2448)$ & .559 \\
\hline$>20 \%$ & $38(1-852)$ & $30(0-813)$ & $65(4-1008)$ & .295 \\
\hline$>25 \%$ & $11(0-264)$ & $7(0-264)$ & $16(0-335)$ & .425 \\
\hline$>30 \%$ & $0(0-101)$ & $0(0-65)$ & $1.0(0-149)$ & .364 \\
\hline \multicolumn{5}{|l|}{$\mathrm{kSO}_{2}$} \\
\hline$<70 \%$ & $46(0-479)$ & $30(0-332)$ & $194(25-817)$ & .005 \\
\hline$<65 \%$ & $0(0-39)$ & $0(0-32)$ & $16(0-103)$ & .001 \\
\hline$<60 \%$ & $0(0-1)$ & $0(0-0)$ & $0(0-9)$ & .001 \\
\hline$<55 \%$ & $0(0-1)$ & $0(0-0)$ & $0(0-1)$ & .013 \\
\hline \multicolumn{5}{|c|}{ Decline from baseline } \\
\hline$>5 \%$ & $3693(326-8782)$ & $3437(321-8425)$ & $3839(430-14634)$ & .382 \\
\hline$>10 \%$ & $1085(13-3803)$ & $942.0(5-3482)$ & $1322.5(46-8614)$ & .168 \\
\hline$>15 \%$ & $86(0-896)$ & $67(0-684)$ & $214(5-4221)$ & .050 \\
\hline$>20 \%$ & $3(0-95)$ & $0(0-60)$ & $18(0-1133)$ & .013 \\
\hline$>25 \%$ & $0(0-15)$ & $0(0-1)$ & $4(0-159)$ & $<.001$ \\
\hline$>30 \%$ & $0(0-5)$ & $0(0-0)$ & $0(0-6)$ & .009 \\
\hline
\end{tabular}

Data are presented as median (interquartile range). $\mathrm{CSA}-\mathrm{AKI}$, Cardiac surgery-associated acute kidney injury; $\mathrm{SSo}_{2}$, brain oxygen saturation; $\mathrm{KSO}_{2}$, kidney oxygen saturation. 
TABLE 5. Univariate and multivariable logistic regression analysis for $\mathrm{kSo}_{2}$ as marker for cardiac surgery-associated acute kidney injury: Unadjusted and adjusted odds ratio (internal validation using bootstrap resampling for 100 replications)

\begin{tabular}{|c|c|c|c|c|c|c|}
\hline & Unadjusted OR $(95 \%$ CI $)$ & $P$ value & $\beta$-Coefficient & SE & Adjusted OR (95\% CI) & $P$ value \\
\hline \multicolumn{7}{|l|}{$\mathrm{kSO}_{2}$} \\
\hline$<70 \%$ & $3.05(1.07-8.70)$ & .037 & 0.807 & 1.751 & $2.24(0.76-6.65)$ & .146 \\
\hline$<65 \%$ & $5.18(2.19-12.25)$ & .000 & 1.371 & 0.547 & $3.94(1.56-9.92)$ & .004 \\
\hline$<60 \%$ & $3.99(1.67-9.49)$ & .002 & 1.104 & 0.540 & $3.02(1.19-7.62)$ & .020 \\
\hline$<55 \%$ & $3.91(1.32-11.53)$ & .014 & 1.187 & 0.688 & $3.28(1.06-10.11)$ & .039 \\
\hline \multicolumn{7}{|c|}{ Decline from baseline } \\
\hline$>5 \%$ & $0.70(0.19-2.57)$ & .591 & -0.478 & 2.90 & $0.62(0.15-2.60)$ & .514 \\
\hline$>10 \%$ & $1.52(0.51-4.51)$ & .453 & 0.379 & 1.506 & $1.46(0.46-4.62)$ & .519 \\
\hline$>15 \%$ & $2.00(0.78-5.16)$ & .152 & 0.562 & 0.825 & $1.75(0.64-4.82)$ & .276 \\
\hline$>20 \%$ & $2.25(0.97-5.22)$ & .058 & 0.992 & 0.530 & $2.70(1.07-6.81)$ & .036 \\
\hline$>25 \%$ & $3.90(1.68-9.02)$ & .002 & 1.280 & 0.459 & $3.60(1.46-8.85)$ & .005 \\
\hline$>30 \%$ & $3.18(1.25-8.08)$ & .015 & 1.053 & 0.564 & $2.86(1.07-7.70)$ & .036 \\
\hline Age, $y$ & $1.04(0.991 .09)$ & .167 & 0.065 & 0.032 & $1.07(1.00-1.14)$ & .042 \\
\hline CPB time, $\min$ & $1.02(1.01-1.03)$ & .002 & 0.016 & 0.005 & $1.02(1.00-1.03)$ & .003 \\
\hline
\end{tabular}

$O R$, Odds ratio; $C I$, confidence interval; $S E$, standard error; $\mathrm{KSO}_{2}$, kidney oxygen saturation; $C P B$, cardiopulmonary bypass.

CSA-AKI. Fourth, multivariable logistic regression revealed that age and CPB time significantly correlated with the development of postoperative CSA-AKI.

Some studies have examined the relationship between $\mathrm{kSO}_{2}$ with NIRS and the development of CSA-AKI during surgery, but few studies have analyzed their relationship during more than 24 hours after the intervention. ${ }^{10,16}$ Unlike in our study, the study by Choi and colleagues ${ }^{10}$ demonstrated that postoperative CSA-AKI was significantly related to intraoperative $\mathrm{kSO}_{2}$ desaturation, but not during the postoperative extent. This study demonstrates monitoring $\mathrm{rSo}_{2}$ during the procedure is not enough; $\mathrm{rSo}_{2}$ must also be recorded after cardiac surgery. Desaturations observed during the postoperative course, but not during the intraoperative phase, were related to the development of postoperative CSA-AKI. This may be supported by our findings that there was no statistically significant difference between the MAP or peak lactate levels and postoperative CSA-AKI during the intraoperative interval $(P=.819$ and $P=.256$, respectively). Both non-CSA-AKI and
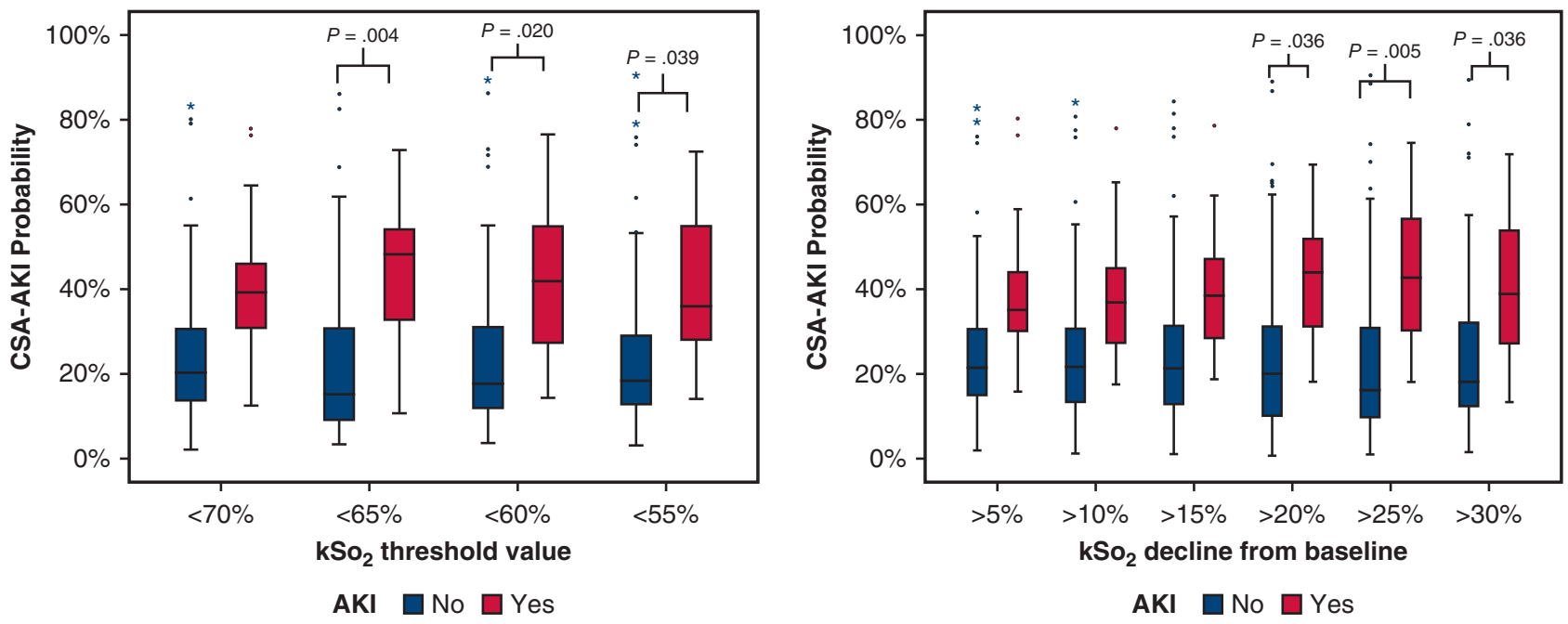

A

B

FIGURE 1. Box plot of the probability of postoperative CSA-AKI at each $\mathrm{kSo}_{2}$ threshold value (A) and at multiple declines from baseline (B). The upper and lower borders of the box represent the upper and lower quartiles. The middle horizontal line represents the median. The upper and lower whiskers represent the maximum and minimum values of non-outliers. Extra dots represent outliers. $C S A-A K I$, Cardiac surgery-associated acute kidney injury; $k S o_{2}$, kidney oxygen saturation; $A K I$, acute kidney injury. 


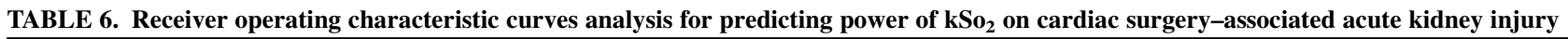

\begin{tabular}{|c|c|c|c|c|c|c|}
\hline & AUC & SE & CI $(95 \%)$ & Sensitivity & Specificity & $P$ value \\
\hline \multicolumn{7}{|c|}{ Absolute threshold } \\
\hline$<70 \%$ & 0.660 & 0.054 & $0.555-0.766$ & 85.7 & 33.7 & .006 \\
\hline$<65 \%$ & 0.679 & 0.054 & $0.573-0.785$ & 71.4 & 67.4 & .002 \\
\hline$<60 \%$ & 0.652 & 0.059 & $0.535-0.768$ & 45.7 & 82.6 & .010 \\
\hline$<55 \%$ & 0.588 & 0.061 & $0.469-0.708$ & 27.4 & 91.9 & .013 \\
\hline \multicolumn{7}{|c|}{ Decline from baseline } \\
\hline$>5 \%$ & 0.552 & 0.063 & $0.428-0.676$ & 88.2 & 8.5 & .382 \\
\hline$>10 \%$ & 0.581 & 0.061 & $0.462-0.701$ & 85.3 & 20.7 & .170 \\
\hline$>15 \%$ & 0.611 & 0.060 & $0.493-0.729$ & 79.4 & 34.1 & .061 \\
\hline$>20 \%$ & 0.639 & 0.059 & $0.523-0.755$ & 67.6 & 51.9 & .019 \\
\hline$>25 \%$ & 0.679 & 0.058 & $0.566-0.793$ & 58.8 & 73.2 & .002 \\
\hline$>30 \%$ & 0.609 & 0.061 & $0.490-0.728$ & 35.3 & 85.4 & .006 \\
\hline
\end{tabular}

$A U C$, Area under the curve; $S E$, standard error; $C I$, confidence interval.

CSA-AKI groups maintained a MAP greater than $50 \mathrm{~mm}$ $\mathrm{Hg}$ during the surgery. The optimal MAP to prevent CSAAKI during $\mathrm{CPB}$ is unknown. Almost all studies that examine MAP during CPB have demonstrated conflicting data. Azau and colleagues ${ }^{17}$ demonstrated that increasing MAP does not reduce CSA-AKI.

There are many factors that still contribute to the appearance of CSA-AKI even after surgery that deserve to be detected and treated, such as low cardiac output syndrome, systemic inflammatory response, adrenergic discharge, vasoplegia, anemia, excessive bleeding, sepsis, and nephrotoxins (eg, antibiotics, contrast media, angiotensin-converting enzyme inhibitors, cephalosporins, aminoglycosides, nonsteroidal anti-inflammatory drugs), all of which impair $\mathrm{kSO}_{2}$ and contribute to the development CSA-AKI. Thus, it is reasonable to continuously monitor $\mathrm{kSo}_{2}$ to allow for the optimal treatment of the renal condition.

During and after cardiac surgery, there is a state of hemodynamic and systemic oxygenation impairment. Recent guidelines emphasize achieving adequate tissue perfusion and systemic oxygen supply-demand metabolism guided by a strategy called "goal-directed therapy" to monitor direct and indirect parameters such as cardiac index, lactate, $\mathrm{MAP}$, and venous oxygen saturation $\left(\mathrm{Svo}_{2}\right)$, which has been shown to improve outcomes after cardiac surgery. However, this strategy only reflects global parameters of systemic perfusion. The regional tissue perfusion and oxygenation levels are different according to different organs, (eg, oxygen consumption is the greatest in the heart than in any other organ). ${ }^{18}$ NIRS tries to overcome these limitations of indirect perfusion and oxygenation parameters, and NIRS provides a noninvasive tool for goal-directed therapy providing real-time assessment of regional oxygen saturation. It can detect important alterations in critical tissue bed perfusions, including the kidney, which results in organ injury. ${ }^{19}$

NIRS is commonly used to monitor $\mathrm{bSo}_{2}$ during cardiac surgery. ${ }^{20}$ According to Slater and colleagues, ${ }^{9}$ intraoperative $\mathrm{bSo}_{2}$ desaturation is associated with poor neurologic outcomes, such as stroke, neurologic dysfunction, and prolonged hospital stays. ${ }^{20,21}$ However, NIRS is not usually placed to monitor kidney function during adult cardiac surgery or the postoperative time span. No correlation was shown between $\mathrm{bSo}_{2}$ with the $\mathrm{kSo}_{2}$ during the postoperative stage, probably because of cerebral autoregulation of the blood flow that allows relatively constant brain blood flow in case of poor systemic perfusion pressure. ${ }^{22}$ Similar to our observations, several studies have shown variable NIRS correlations to oxygen saturation parameters in different organs or no correlation at all. ${ }^{20,23-25}$ Recently, Olbrecht and colleagues ${ }^{26}$ demonstrated that $\mathrm{bSo}_{2}$ remains unchanged in low arterial pressure events, suggesting that the brain oxygen supply-demand relationship is preserved in these cases or these low arterial pressures are within the cerebral autoregulation parameters for blood flow. ${ }^{26}$ Alterations in $\mathrm{rSo}_{2}$ may reflect local changes but may not correlate to other organ oxygen saturation or hypoperfusion. ${ }^{27}$ Therefore, it is essential to have a somatic monitorization, as demonstrated in this study.

Multivariable logistic regression analysis revealed advanced age and long CPB time significantly correlated to postoperative CSA-AKI. These findings are supported by the current data, which report the etiology of postoperative CSA-AKI as multifactorial. ${ }^{28}$ First, age is a recognized independent risk factor for postoperative CSA-AKI. It is even a consistent variable in various risk models for predicting CSA-AKI, such as the Society of Thoracic Surgeons score, EuroSCORE, and the Age, Creatinine, Ejection Fraction score. ${ }^{29}$ Second, CPB implies a pathophysiologic state that entails a series of factors: activation of a systemic inflammatory response, complement system activation, coagulopathy, nonpulsatile blood flow that produces changes in kidney vasomotor tone and renal ischemia, microemboli generation, and hemolysis. This situation is magnified as long as the duration of the CPB time increases. ${ }^{30}$ Moreover, there is a differential impact of the type of surgery on postoperative CSA-AKI. In fact, the association between 

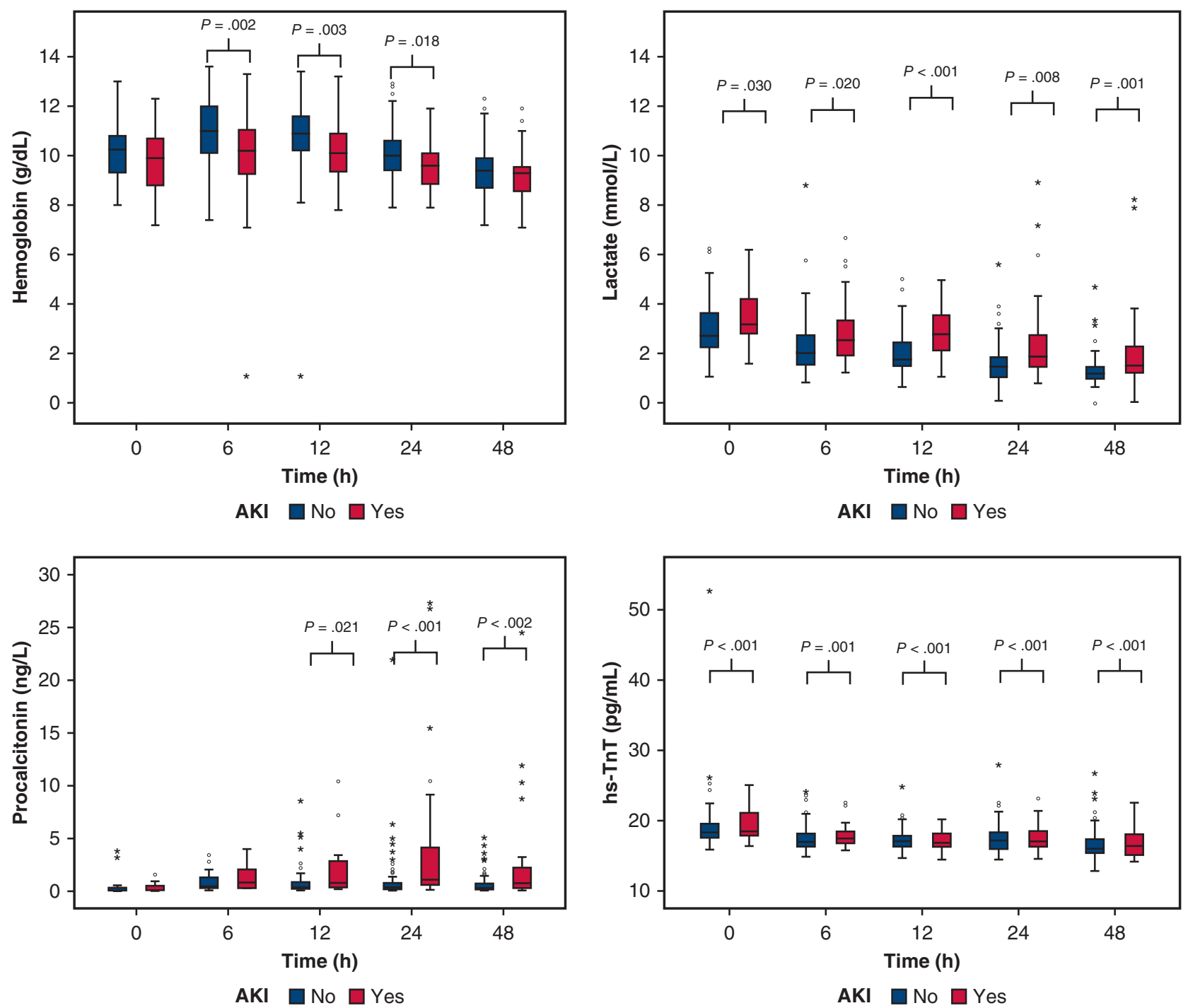

FIGURE 2. Box plot of postoperative laboratory findings according to the presence of CSA-AKI. The upper and lower borders of the box represent the upper and lower quartiles. The middle horizontal line represents the median. The upper and lower whiskers represent the maximum and minimum values of nonoutliers. Extra dots represent outliers. AKI, Acute kidney injury; $h s-T n T$, high-sensitivity troponin T.

combined operations (ie, coronary artery bypass grafting and valvular surgery) and greater rates of AKI is not surprising and has been demonstrated in addition to the support provided by this study. ${ }^{28}$ Depending on its multiple modifications in definitions and classifications, the incidence of CSA-AKI widely varies from $7 \%$ to $40 \%$ according to previous studies. ${ }^{31-33}$ The incidence of postoperative CSAAKI in this study was $28.9 \%$, results similar to the currently reported data and may be related to the application of strategies focused on reducing postoperative CSA-AKI, such as ensuring an adequate perfusion pressure, optimizing cardiac output, enhancing hydroelectric replenishment, and avoiding nephrotoxic drugs.

Validated critical levels for $\mathrm{kSO}_{2}$ are lacking. In fact, the definition of pathological NIRS measurements is not consistent among studies, ranging from $50 \%$ to $80 \%$, and those studies do not account for the magnitude and duration of desaturations. ${ }^{23}$ Area decline from baseline seems to be a better parameter than isolated NIRS measurements, such as minimum $\mathrm{rSo}_{2}$ values or different percentages of desaturations, because it takes into account not only the magnitude but also the duration of that desaturation.

Considering the peak of $\mathrm{SCr}$ might be observed up to 7 days after surgery (according to the definition of KDIGO Criteria for CSA-AKI) and the NIRS measurements were only registered during the first 48 hours postoperatively, $\mathrm{kSo}_{2}$ measurements provide a time-limited lapse to take prompt responses. This finding is the main benefit and the greatest significant advantage offered by this new technique. It not only improves the current early identification 

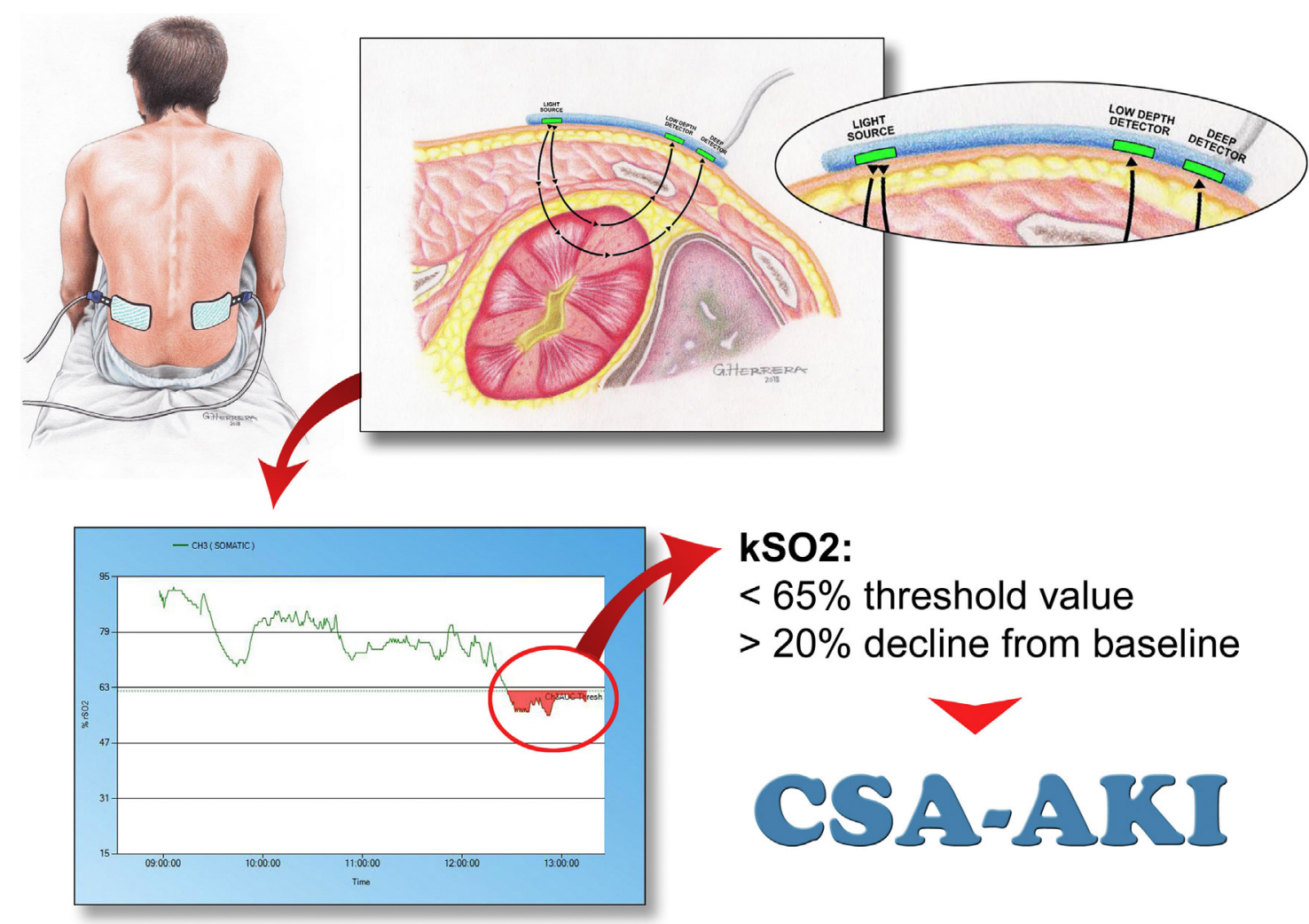

kSO2:

$<65 \%$ threshold value

$>20 \%$ decline from baseline

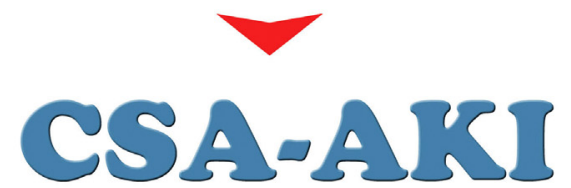

FIGURE 3. $\mathrm{kSO}_{2}$ desaturation during the postoperative extent significantly increases the risk of CSA-AKI. NIRS sensors, which consisted of 1 emitter and 2 receivers, being the farthest for deep detection, were positioned on both flank zones corresponding to the underlying kidneys, previously identified using ultrasound guidance to monitor the $\mathrm{kSo}_{2}$. This was registered every 30 seconds during the entire intraoperative and postoperative extent, until 48 hours after cardiac surgery, resulting in a graph in which the dynamic changes of $\mathrm{kSO}_{2}$ values per unit of time were represented. $\mathrm{kSO}_{2}$ was analyzed in terms of area measurements $(\%$-min) from multiple absolute threshold values. A desaturation underneath the absolute threshold value of $65 \%$ or a decline greater than $20 \%$ from baseline value in the $\mathrm{kSo}_{2}$ during the postoperative period is significantly associated with CSA-AKI; thus, by avoiding these desaturation levels, the prevention of CSA-AKI could be considerably enhanced. AUC, Area under the curve; $C I$, confidence interval; $\mathrm{kSo}_{2}$, kidney oxygen saturation.

but also provides a noninvasive, real-time marker, which can serve as a parameter to prevent postoperative CSAAKI. Whenever $\mathrm{kSo}_{2}$ falls below the $65 \%$ threshold value or declines greater than $20 \%$ from the baseline value, it cannot last for more than 2.2 minutes.

$\mathrm{SCr}$ offers little and delayed information concerning kidney perfusion and oxygen delivery. In contrast, $\mathrm{KSo}_{2}$ provides continuous, real-time, noninvasive assessment of kidney oxygen supply-demand balance, which correlates to the kidney regional perfusion. This study has demonstrated $\mathrm{kSO}_{2}$ to reliably detect CSA-AKI in an earlier manner than $\mathrm{SCr}$, and moreover its measures are suitable targets for a goal-directed therapy, allowing a time-limited span to take prompt responses or treat deficiencies in kidney perfusion before irreversible renal failure may occur.

Urinary biomarkers like $\mathrm{kSO}_{2}$ distinguished outcomes in the early postoperative term after pediatric cardiac surgery. ${ }^{32}$ However, unlike biomarkers, which cannot be measured continuously and generally require a substantial amount of money and time for their assay and analysis, NIRS enables noninvasive, real-time monitoring. ${ }^{10}$ The addition of $\mathrm{kSO}_{2}$ to biomarkers could allow for real-time monitoring and improve CSA-AKI detection. Furthermore, NIRS monitoring would be useful in elderly patients with a long CPB time to detect CSA-AKI early. $\mathrm{kSO}_{2}$ should not decrease less than $65 \%$ or decline greater than $20 \%$ from the baseline value during the postoperative time-lapse, especially in elderly patients with longer CPB times.

It may be feasible to introduce this method for the early identification of postoperative AKI, considering it can reduce costs despite the equipment and the sensor costs. The early identification of postoperative AKI through NIRS measurements can reduce the prolonged ICU and hospital length of stay related to postoperative CSA-AKI. In a cost analysis study, Walsh and colleagues ${ }^{34}$ demonstrated a significant saving when using INVOS in the management of both mixed and coronary artery bypass graftonly cardiac surgery. Furthermore, Murkin and colleagues ${ }^{35}$ 


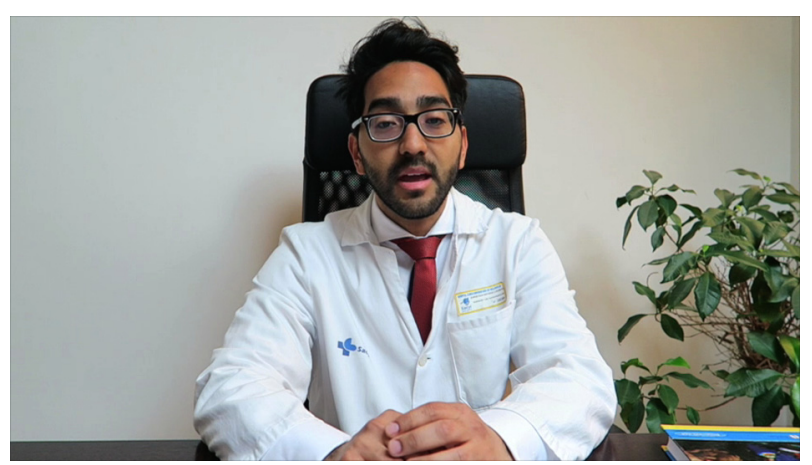

VIDEO 1. Dr Ortega-Loubon explains the relevance and importance of the article. Absolute kidney oxygen desaturations less than $65 \%$ or decreases greater than $20 \%$ from baseline value during the postoperative stage are novel markers for developing postoperative CSA-AKI. Therefore, kidney oxygen saturation monitoring could be a real-time, noninvasive strategy to improve CSA-AKI detection, allowing prompt and appropriate responses. Video available at: https://www.jtcvs.org/article/S0022-5223(18)32785-5/fulltext.

demonstrated that treatment of declines in $\mathrm{rSo}_{2}$ is associated with a shorter ICU length of stay and, therefore, a reduction in the cost of care.

\section{Study Limitations}

First, this was an exploratory study, and multiple threshold values were tested, leading to a potential type 1 error. Although an internal validation method was performed, these threshold values ideally would be tested in a different patient population. Likewise, although ORs were sizable and statistically significant, the accuracy according to AUC-ROC analysis was modest. Second, it is a single-center study with a relatively small sample of patients, although similar studies were reported with a similar number of patients. Severe $\mathrm{kSO}_{2}$ desaturations were uncommon. Nevertheless, the number of events was sufficient to conduct a regression analysis. A large multicenter study with a heterogeneous population and with more severe $\mathrm{kSo}_{2}$ desaturation events would be desirable. Also, a costeffectiveness study addressing the feasibility of introducing this new strategy in daily care should be considered. Last, the available NIRS sensor can only measure the regional level of tissue oxygen saturation 3 to $4 \mathrm{~cm}$ beneath the local tissue area. As a result, we had to exclude those patients with renal depths $4 \mathrm{~cm}$ or greater. The median renal depths for the study population were $1.7 \mathrm{~cm}$ for the right kidney and $1.9 \mathrm{~cm}$ for the left kidney. A sensor that permits deeper monitoring is necessary to include patients with deeper renal depths like those from the general population, given that obesity is a common morbidity. Despite these limitations, this study provides a useful insight into the incidence of postoperative CSA-AKI and its relationship with postoperative $\mathrm{bSo}_{2}$ and $\mathrm{kSO}_{2}$ in adult cardiac surgery.

\section{CONCLUSIONS}

Continuous kidney NIRS monitoring might be a promising noninvasive tool for predicting AKI during the postoperative period of adult cardiac surgery because postoperative $\mathrm{KSO}_{2}$ monitoring is related to the development of AKI after adult cardiac surgery (Video 1). Our findings suggest that $\mathrm{kSO}_{2}$ should not decrease below the threshold value of $65 \%$ or decline greater than $20 \%$ from the baseline value during the postoperative time to prevent CSA-AKI. In addition, it is a well-known complication of paramount importance related to higher mortality rates, as demonstrated in this study, in which, despite the small number of patients needed to obtain a statistically significant result, the only 2 deaths registered were in the CSA-AKI group-1 of which occurred during the first 30 days after surgery. Because postoperative $\mathrm{kSO}_{2}$ desaturation is related to the development of CSA-AKI, it could also be related to death. A large number of patients are required to prove this relationship. It would also be sensible to examine if certain therapeutic measures—such as optimizing cardiac output—can improve $\mathrm{kSo}_{2}$. Further clinical trials are needed to evaluate therapeutic measures for maintaining a normal $\mathrm{kSO}_{2}$ compared with the standard therapy and to determine the influencing factors on the development of CSA-AKI.

\section{Conflict of Interest Statement}

Authors have nothing to disclose with regard to commercial support.

\section{References}

1. Zakkar M, Bruno VD, Guida G, Angelini GD, Chivasso P, Suleiman MS, et al. Postoperative acute kidney injury defined by RIFLE criteria predicts early health outcome and long-term survival in patients undergoing redo coronary artery bypass graft surgery. J Thorac Cardiovasc Surg. 2016;152:235-42.

2. Mao H, Katz N, Ariyanon W, Blanca-Martos L, Adybelli Z, Giuliani A, et al. Cardiac surgery-associated acute kidney injury. Cardiorenal Med. 2013;3:178-99.

3. Najafi M. Serum creatinine role in predicting outcome after cardiac surgery beyond acute kidney injury. World J Cardiol. 2014;6:1006-21.

4. Martensson J, Martling CR, Bell M. Novel biomarkers of acute kidney injury and failure: clinical applicability. Br J Anaesth. 2012;109:843-50.

5. Ristikankare A, Poyhia R, Kuitunen A, Skrifvars M, Hammainen P, Salmenpera M, et al. Serum cystatin C in elderly cardiac surgery patients. Ann Thorac Surg. 2010;89:689-94.

6. Ortega-Loubon C, Fernandez-Molina M, Carrascal-Hinojal Y, FulquetCarreras E. Cardiac surgery-associated acute kidney injury. Ann Card Anaesth. 2016;19:687-98.

7. Buratto E, Horton S, Konstantinov IE. So near, yet so far: is isolated cerebral near-infrared spectroscopy in neonates nearly as useful as it is noninvasive? $J$ Thorac Cardiovasc Surg. 2017;154:1054-5.

8. Biedrzycka A, Lango R. Tissue oximetry in anaesthesia and intensive care. Anaesthesiol Intensive Ther. 2016;48:41-8.

9. Slater JP, Guarino T, Stack J, Vinod K, Bustami RT, Brown JM III, et al. Cerebral oxygen desaturation predicts cognitive decline and longer hospital stay after cardiac surgery. Ann Thorac Surg. 2009;87:36-45.

10. Choi DK, Kim WJ, Chin JH, Lee EH, Don Hahm K, Yeon Sim J, et al. Intraoperative renal regional oxygen desaturation can be a predictor for acute kidney injury after cardiac surgery. J Cardiothorac Vasc Anesth. 2014;28:564-71.

11. Fuentes-Garcia D, Carceles-Baron MD, Lopez-Lopez R, Roques-Escolar V. Non-invasive oximetry for early detection of cerebral and somatic ischaemia during corrective surgery for aortic coarctation in paediatric patients. Br J Anaesth. 2010;104:780-1. 
12. Abadeer AI, Kurlansky P, Chiuzan C, Truby L, Radhakrishnan J, Garan R, et al. Importance of stratifying acute kidney injury in cardiogenic shock resuscitated with mechanical circulatory support therapy. J Thorac Cardiovasc Surg. 2017; 154:856-64.e4.

13. Chan JL, Kobashigawa JA, Aintablian TL, Dimbil SJ, Perry PA, Patel JK, et al. Characterizing predictors and severity of vasoplegia syndrome after heart transplantation. Ann Thorac Surg. 2018;105:770-7.

14. Tamayo E, Alvarez FJ, Martinez-Rafael B, Bustamante J, Bermejo-Martin JF, Fierro I, et al. Ventilator-associated pneumonia is an important risk factor for mortality after major cardiac surgery. J Crit Care. 2012;27:18-25.

15. Tomsic A, Schotborgh MA, Manshanden JS, Li WW, de Mol BA. Coronary artery bypass grafting-related bleeding complications in patients treated with dual antiplatelet treatment. Eur J Cardiothorac Surg. 2016;50:849-56.

16. Hori D, Hogue C, Adachi H, Max L, Price J, Sciortino C, et al. Perioperative optimal blood pressure as determined by ultrasound tagged near infrared spectroscopy and its association with postoperative acute kidney injury in cardiac surgery patients. Interact Cardiovasc Thorac Surg. 2016;22:445-51.

17. Azau A, Markowicz P, Corbeau JJ, Cottineau C, Moreau X, Baufreton C, et al. Increasing mean arterial pressure during cardiac surgery does not reduce the rate of postoperative acute kidney injury. Perfusion. 2014;29: 496-504.

18. Kopp R, Dommann K, Rossaint R, Schalte G, Grottke O, Spillner J, et al. Tissue oxygen saturation as an early indicator of delayed lactate clearance after cardiac surgery: a prospective observational study. BMC Anesthesiol. 2015;15:158.

19. Tweddell JS, Ghanayem NS, Hoffman GM. Pro: NIRS is "standard of care" for postoperative management. Semin Thorac Cardiovasc Surg Pediatr Card Surg Annu. 2010;13:44-50.

20. Murkin JM, Arango M. Near-infrared spectroscopy as an index of brain and tissue oxygenation. Br J Anaesth. 2009;103(Suppl 1):i3-13.

21. Selnes OA, Gottesman RF, Grega MA, Baumgartner WA, Zeger SL, McKhann GM. Cognitive and neurologic outcomes after coronary-artery bypass surgery. N Engl J Med. 2012;366:250-7.

22. Cipolla MJ. The Cerebral Circulation. San Rafael, CA: Morgan \& Claypool Life Sciences; 2009.

23. Ruf B, Bonelli V, Balling G, Horer J, Nagdyman N, Braun SL, et al. Intraoperative renal near-infrared spectroscopy indicates developing acute kidney injury in infants undergoing cardiac surgery with cardiopulmonary bypass: a case-control study. Crit Care. 2015;19:27.

24. Hoffmann GM, Stuth EA, Jaquiss RD, Vanderwa IPL, Staudt SR, Troshynski TJ, et al. Changes in cerebral and somatic oxygenation during stage 1 palliation of hypoplastic left heart syndrome using continuous regional cerebral perfusion. $J$ Thorac Cardiovasc Surg. 2004;127:223-33.
25. Bhalala U, Nishisaki A, McQueen D, Bird GL, Morrison WE, Nadkarni VM, et al. Change in regional (somatic) near-infrared spectroscopy is not a useful indictor of clinically detectable low cardiac output in children after surgery for congenital heart defects. Pediatr Crit Care Med. 2012;13:529-34

26. Olbrecht VA, Skowno J, Marchesini V, Ding L, Jiang Y, Ward CG, et al. An international, multicenter, observational study of cerebral oxygenation during infant and neonatal anesthesia. Anesthesiology. 2018;128:85-96.

27. Hirsch JC, Charpie JR, Ohye RG, Gurney JG. Near infrared spectroscopy (NIRS should not be standard of care for postoperative management. Semin Thorac Cardiovasc Surg Pediatr Card Surg Annu. 2010;13:51-4.

28. Saydy N, Mazine A, Stevens LM, Jeamart H, Demers P, Page P, et al. Differences and similarities in risk factors for postoperative acute kidney injury between younger and older adults undergoing cardiac surgery. J Thorac Cardiovasc Surg. 2018;155:256-65.

29. Chen SW, Chang CH, Fan PC, Chen YC, Chu PH, Chen TH, et al. Comparison of contemporary preoperative risk models at predicting acute kidney injury after isolated coronary artery bypass grafting: a retrospective cohort study. BMJ Open. 2016;6:e010176.

30. Jorge-Monjas P, Bustamante-Munguira J, Lorenzo M, Heredia-Rodriguez M, Fierro I, Gomez-Sanchez E, et al. Predicting cardiac surgery-associated acute kidney injury: the CRATE score. J Crit Care. 2016;31:130-8.

31. Machado MN, Nakazone MA, Maia LN. Prognostic value of acute kidney injury after cardiac surgery according to kidney disease: improving global outcomes definition and staging (KDIGO) criteria. PLoS One. 2014;9.

32. Hazle MA, Gajarski RJ, Aiyagari R, Yu S, Abraham A, Donohue J, et al. Urinary biomarkers and renal near-infrared spectroscopy predict intensive care unit outcomes after cardiac surgery in infants younger than 6 months of age. $J$ Thorac Cardiovasc Surg. 2013;146:861-7.e1.

33. Gaffney AM, Sladen RN. Acute kidney injury in cardiac surgery. Curr Opin Anaesthesiol. 2015;28:50-9.

34. Walsh D, Bennett M, Bennett S. Cost analysis of patients undergoing cardiac surgery managed with or without cerebral oximetry (INVOS). BMC Proc. 2012;6: O16.

35. Murkin JM, Adams SJ, Novick RJ, Quantz M, Bainbridge D, Iglesias I, et al Monitoring brain oxygen saturation during coronary bypass surgery: a randomized, prospective study. Anesth Analg. 2007;104:51-8.

Key Words: cardiac surgery, acute renal failure, near infrared spectroscopy, prevention, perioperative care 

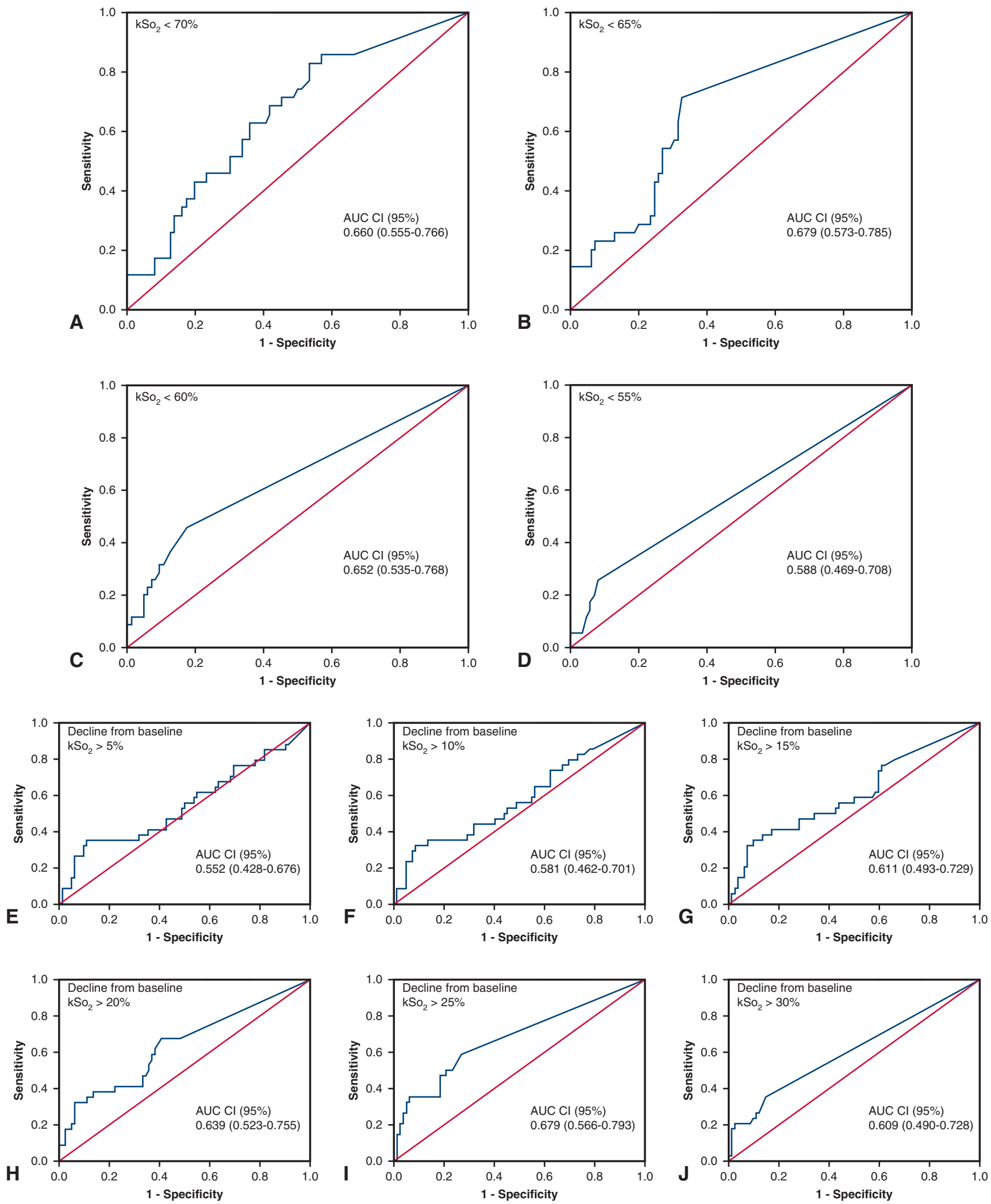

FIGURE E1. AUC-ROC curves at every threshold value (A-D) and every decline from baseline value (E-J). AUC-ROC, Area under the curve-receiver operating characteristic; $\mathrm{kSO}_{2}$, kidney oxygen saturation. 


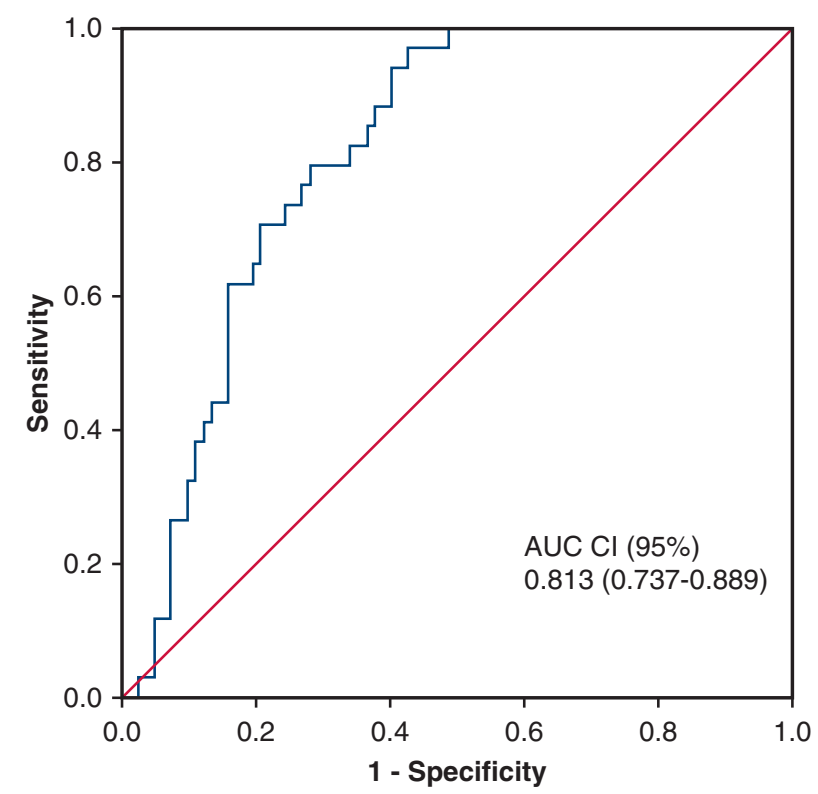

FIGURE E2. AUC-ROC curve. Multivariable logistic regression model for decline of $\mathrm{kSO}_{2}$ greater than $25 \%$. AUC-ROC, Area under the curvereceiver operating characteristic; $k \mathrm{Ko}_{2}$, kidney oxygen saturation.

TABLE E1. Departure logistic regression (forward stepwise)*

\begin{tabular}{lccccrr}
\hline \multicolumn{1}{c}{ CSA-AKI } & B & SE & Wald & df & P & OR (95\% CI) \\
\hline Intercept & -7.366 & 2.595 & 8.060 & 1 & .005 & \\
CPB time (min) & 0.017 & 0.005 & 10.119 & 1 & .001 & $1.017(1.007-1.028)$ \\
Age & 0.067 & 0.034 & 3.818 & 1 & .051 & $1.069(1.000-1.143)$ \\
Decline $>20 \%$ & 1.001 & 0.524 & 3.654 & 1 & .056 & $2.725(0.975-7.576)$ \\
\hline
\end{tabular}

$C S A-A K I$, Cardiac surgery-associated acute kidney injury; $S E$, standard error; $d f$, degrees of freedom; $O R$, odds ratio; $C I$, confidence interval; $C P B$, cardiopulmonary bypass. *Variables: gender; hypertension; $\mathrm{kSo}_{2}<70 \%, \mathrm{kSo}_{2}<65 \%, \mathrm{kSo}_{2}<60 \%, \mathrm{kSo}_{2}<55 \%, \mathrm{kSo}_{2}$ decline $>5 \% ; \mathrm{kSo}_{2}$ decline $>10 \% ; \mathrm{kSo}_{2}$ decline $>15 \%$; $\mathrm{kSo}_{2}$ decline $>20 \%$; $\mathrm{kSo}_{2}$ decline $>25 \% ; \mathrm{kSO}_{2}$ decline $>30 \%$; chronic obstructive pulmonary disease; coronary artery disease; age; body mass index; left ventricular ejection fraction; lactate; procalcitonin; and CPB time.

TABLE E2. Logistic regression (forward stepwise) repeated with the variables that had been significant in the previous analysis

\begin{tabular}{lcccccr}
\hline & \multicolumn{3}{c}{ Parameter estimates } & df & P & OR (95\% CI) \\
\hline \multicolumn{1}{c}{ CSA-AKI } & B & SE & Wald & df & \\
\hline Intercept & -7.577 & 2.435 & 9.682 & 1 & .002 & $1.016(1.006-1.027)$ \\
CPB time (min) & 0.016 & 0.005 & 10.096 & 1 & .001 & $1.076(1.011-1.146)$ \\
Age & 0.074 & 0.032 & 5.246 & 1 & .022 & .036 \\
Decline $>20 \%$ & 0.992 & 0.472 & 4.408 & 1 & $2.697(1.068-6.807)$ \\
\hline
\end{tabular}

$C S A-A K I$, Cardiac surgery-associated acute kidney injury; $S E$, standard error; $d f$, degrees of freedom; $O R$, odds ratio; $C I$, confidence interval; $C P B$, cardiopulmonary bypass. 
TABLE E3. Bootstrap validation for the logistic regression model

\begin{tabular}{|c|c|c|c|c|c|c|}
\hline \multicolumn{7}{|c|}{ Bootstrap for parameter estimates } \\
\hline \multirow[b]{3}{*}{ CSA-AKI } & \multirow[b]{3}{*}{ B } & \multicolumn{5}{|c|}{ Bootstrap* } \\
\hline & & \multirow[b]{2}{*}{ Bias } & \multirow[b]{2}{*}{ SE } & \multirow[b]{2}{*}{$\boldsymbol{P}$} & \multicolumn{2}{|c|}{$95 \% \mathrm{CI}$} \\
\hline & & & & & Lower & Upper \\
\hline Intercept & -7.577 & -0.755 & 2.618 & .002 & -14.114 & -4.141 \\
\hline CPB time (min) & 0.016 & 0.001 & 0.007 & .003 & 0.006 & 0.034 \\
\hline Age & 0.074 & 0.008 & 0.035 & .007 & 0.025 & 0.157 \\
\hline Decline $>20 \%$ & 0.992 & -0.011 & 0.521 & .032 & -0.004 & 2.052 \\
\hline
\end{tabular}

CSA-AKI, Cardiac surgery-associated acute kidney injury; $S E$, standard error; $d f$, degrees of freedom; $C I$, confidence interval; $C P B$, cardiopulmonary bypass. *Unless otherwise noted, bootstrap results are based on 100 bootstrap samples. The rest of the saturation/desaturation levels were analyzed by performing the bootstrapping for each model with only 3 variables: age, $\mathrm{CPB}$, and the dichotomous variable corresponding to the saturation level. The results are shown in the article (Table 5). 